\title{
RECURSO COMPUTACIONAL AUXILIAR AO ENSINO DO RACIOCÍNIO DIAGNÓSTICO: INTENÇÕES E VALORES IDENTIFICADOS*
}

Fabiane Novais Moreno', Marcia Regina $\mathrm{Cubas}^{2}$, Andreia Malucelli³ ${ }^{3}$ Carla Luiza da Silva ${ }^{4}$

RESUMO: Trata-se de pesquisa exploratória qualitativa, cujo objetivo foi analisar intenções e valores de um recurso computacional para auxílio ao ensino do processo de raciocínio diagnóstico. Após aprovação por Comitê de Ética, foram entrevistados seis docentes de enfermagem, cujos discursos foram submetidos à análise de discurso. Os temas foram agrupados em nove subcategorias, sendo cinco relacionadas às intenções: facilitador de processo de raciocínio clínico, auto avaliação e avaliação do aluno, reflexão sobre a prática, facilitador do ensino-aprendizagem e indutor de mudança; e quatro aos valores: utilidade para prática assistencial, inovação, aprendizagem ecapacitação contínua. Verificou-se que os docentes assimilaramanecessidade dautilização derecursos computacionais, mas enfatizam que esse somente beneficiará o aluno a desenvolver o raciocínio se o aprendiz realizar estudos paralelos. A análise evidencia que o recurso pode aperfeiçoar o processo de raciocínio diagnóstico e dinamizar o estudo de uma maneira interativa, sem preterirométodo tradicional de ensino DESCRITORES: Informática em enfermagem; Tecnologia educacional; Educação em enfermagem; Aprendizagem.

\section{COMPUTER RESOURCES FOR ASSISTING IN THE TEACHING OF DIAGNOSTIC REASONING: IDENTIFIED INTENTIONS AND VALUES}

ABSTRACT: This is exploratory qualitative research, with the objective of analyzing the intentions and values of a computer resource for assistance in the teaching of the process of diagnostic reasoning. Following approval from the Ethics Committee, six professors of Nursing were interviewed, whose discourses were submitted to discourse analysis. The issues were grouped into nine subcategories, five being related to the intentions: facilitative for the process of clinical reasoning, self-evaluation and evaluation of the student, reflection on practice, facilitative for teaching-learning, and inductive of change; and four to the values: utility for assistential practice, innovation, learning and continuous training. It was ascertained that the professors assimilated the need for computer resources, but emphasize that this only helps the student to develop the reasoning if the student carries out parallel studies. The analysis evidences that the resource can improve the process of diagnostic reasoning and energize study in an interactive way, without disadvantaging the traditional method of teaching. DESCRIPTORS: Information technology in Nursing; Educational technology; Education in Nursing; Learning.

\section{RECURSO COMPUTACIONAL AUXILIAR A LA ENSEÑANZA DEL RACIOCINIO DIAGNÓSTICO: INTENCIONES Y VALORES IDENTIFICADOS}

RESUMEN: Esta es una investigación exploratoria cualitativa, cuyo objetivo fue analizar intenciones y valores de un recurso computacional para ayuda a la enseñanza del proceso de raciocinio diagnóstico. Después de la aprobación por Comité de Ética, fueron entrevistados seis docentes de enfermería, cuyos discursos fueron sometidos al análisis de discurso. Los temas fueron agrupados en nueve subcategorías, siendo cinco asociadas a las intenciones: facilitador de proceso de raciocinio clínico, auto evaluación y evaluación del alumno, reflexión acerca de la práctica, facilitador de la enseñanza aprendizaje e inductor de cambio; y cuatro a los valores: utilidad para práctica asistencial, innovación, aprendizaje y capacitación continua. Se verificó que los docentes comprendieron la necesidad de la utilización de recursos computacionales, pero enfatizan que ese solo beneficiará al alumno a desarrollar el raciocinio si él realizarlo estudios paralelos. El análisis muestra que el recurso puede optimizar el proceso de raciocínio diagnóstico y dinamizar el estúdio de una manera interactiva, sin abstenerse del método de enseñanza tradicional.

DESCRIPTORES: Informática en enfermería; Tecnología educacional; Educación en enfermería; Aprendizaje.

\footnotetext{
*Recorte de dissertação intitulada 'Intenções e valores identificados por docentes no uso de uma ferramenta computacional de auxílio ao desenvolvimento do raciocínio diagnóstico em enfermagem' apresentada ao Programa de Pós-graduação em Tecnologia em Saúde da Pontifícia Universidade Católica do Paraná, em 2013. Parte de pesquisa com fomento do Edital Universal n.14/2009-0 do CNPq.

${ }^{1}$ Enfermeira do Hospital VITA Batel.

${ }^{2}$ Enfermeira. Doutora em Enfermagem. Professora e Coordenadora do Programa de Pós-Graduação em Tecnologia em Saúde da Pontifícia Universidade Católica do Paraná - PUCPR.

${ }^{3}$ Bacharel em Informática. Doutora em Engenharia Eletrotécnica e de Computadores. Professora do Programa de Pós-Graduação em Informática da PUCPR.

${ }^{4}$ Enfermeira. Mestre em Tecnologia em Saúde. Professora da Universidade Estadual de Ponta Grossa.
}

Autor correspondente:

Recebido: 22/05/2013

Marcia Regina Cubas Aprovado: 01/10/2013

Pontifícia Universidade Católica do Paraná

Rua Imaculada Conceição, 1155 - 80215-901 - Curitiba-PR-Brasil

E-mail:m.cubas@pucpr.br 


\section{INTRODUÇÃO}

No Brasil, parte da prática assistencial da enfermagem é organizada pelo Processo de Enfermagem (PE); entre suas fases, a do Diagnóstico de Enfermagem (DE) é destacada como a mais complexa ${ }^{(1)}$. Seu foco está relacionado às respostas humanas aos processos saúde doença; aos tratamentos a que os indivíduos são submetidos; e ao processo de vida nos âmbitos individual, familiar ou da coletividade. Para registro do $\mathrm{DE}$, os enfermeiros utilizam linguagens próprias da profissão, dentre elas, a Classificação Internacional para a Prática de Enfermagem (CIPE®), cujo uso requer o raciocínio clínico para determinar necessidades de cuidado, pois, na maioria das vezes, as manifestações apresentadas são diferentes da literatura, sendo importante que se possibilite, durante a graduação, intercâmbios entre a prática e a teoria ${ }^{(1-2)}$.

É imperativo que professores aperfeiçoem seus métodos de ensino para o desenvolvimento do raciocínio clínico dos alunos. Por outro lado, docentes são desafiados pelas diferentes formas de ensino-aprendizagem que são mediadas por tecnologias como instrumento educacional $^{(3-4)}$. E, ao incluí-las em sua prática, eles podem apresentar dificuldades para se adaptar e fazer diferente o que sempre fizeram ${ }^{(3)}$. Soma-se a esta problemática, a necessidade de que a construção do conhecimento aconteça de forma que se presencie troca de experiências entre professor e aluno, sendo o aluno responsável pela construção do seu próprio conhecimento, sem dependência direta do professor ${ }^{(4-5)}$. Uma das possibilidades para que isso ocorra é o uso de tecnologias capazes de colocar o professor como um facilitador e não um condutor do processo de ensino ${ }^{(4-5)}$.

Entre as tecnologias, a informática educativa é um instrumento que permite o uso de simulações que favorece ao aluno praticar situações, abstratas ou reais, para as quais ainda não esteja preparado ${ }^{(6-7)}$, estimulando a participação ativa e possibilitando flexibilidade do processo ensino-aprendizagem ${ }^{(8)}$. Docentes de Enfermagem relatam que as tecnologias digitais devem ser assimiladas, mas apontam a necessidade de ampla reflexão sobre as intenções e os valores incorporados a elas ${ }^{(9)}$. Esta reflexão poderá diminuir a vulnerabilidade decorrente da aceitação de que a implantação de uma tecnologia resolva os problemas da enfermagem, estabelecendo um recurso compatível com a prática do cuidado humano, em que o professor possui o papel de facilitador no processo de aprendizagem ${ }^{(9)}$.

Esta pesquisa teve como objetivo analisar as inten- ções e os valores de um recurso computacional para auxílio ao ensino do processo de raciocínio diagnóstico. É parte de uma pesquisa que avalia um protótipo de um sistema computacional para auxiliar o aprendizado do raciocínio diagnóstico individual e coletivo, que faz uso da CIPE®.

O protótipo consiste em uma plataforma com interface aluno e professor e possui como base um método de ensino ${ }^{(10)}$ que usa estudos de caso simulados como apoio para aprendizagem do raciocínio e do uso de sistemas classificatórios. Em linhas gerais, a interface professor possibilita: a inserção de estudo de caso com grau de dificuldade e taxa de acerto para resposta dos alunos; o estabelecimento das necessidades humanas relacionadas aos problemas e potencialidades; a indicação dos principais $\mathrm{DE}$, Resultados e Intervenções

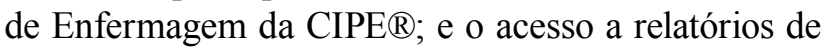
desempenho por aluno e por estudo de caso. A interface do aluno permite: o acesso a diferentes estudos com graus de complexidade distintos; a resolução dos estudos a partir dos problemas e potencialidades encontrados no caso, organizando-os por necessidades humanas; a escolha de DE, Resultados e Intervenções de Enfermagem da CIPE® previamente incluídas pelos professores; e o acesso a relatórios de seu desempenho individual. Cabe ressaltar que a interface do aluno não permite que ele passe para a atividade seguinte sem atingir o percentual de acerto estabelecido pelo professor responsável pelo estudo, bem como disponibiliza ícones com conceitos chaves e links para textos de referência.

\section{MÉTODO}

Trata-se de uma pesquisa exploratória com abordagem qualitativa, tendo como cenário uma Escola de Enfermagem de uma Instituição Comunitária, localizada na capital do Estado do Paraná. Foram incluídos mestres e doutores na área de Enfermagem ou da Educação, que lecionavam disciplinas de prática clínica, ou estágio supervisionado na área hospitalar ou saúde coletiva, por um período superior a três anos. Foram excluídos docentes com duplo vínculo em instituição formadora e que não praticavam o uso de classificações de DE. Participaram da pesquisa seis docentes que atuavam a partir do segundo ano da graduação, sendo seus discursos referenciados pela letra $\mathrm{P}$, com numerações de um a seis.

A coleta de dados, realizada nos meses de junho a dezembro de 2010, se deu por meio de um questionário semiestruturado, composto por cinco questões abertas 
que abordavam aspectos conceituais relacionados às intenções (finalidade da ferramenta e propósito do uso de recurso computacional) e os valores do recurso pedagógico computacional (relação entre a ferramenta e a prática do ensino do raciocínio diagnóstico e a relação entre a ferramenta e a prática do raciocínio diagnóstico pelos alunos e por enfermeiros). As entrevistas foram transcritas literalmente e submetidas à correção de erros gramaticais e vícios de linguagem. Ressalta-se que antes da entrevista o protótipo do sistema foi apresentado aos docentes.

A organização e a análise dos dados seguiu a estruturação da análise de discurso ${ }^{(11)}$. As respostas individuais a cada uma das perguntas foram agregadas para gerar o corpus de análise, organizado por Temas e Figuras ${ }^{(11)}$, que originaram frases temáticas, classificadas nas categorias de análises e em subcategorias interpretativas. As categorias de análise foram: Intenção do Recurso Computacional, entendendo por intenção do que que se pretende fazer; propósito, plano, ideia; o que se procura alcançar, conscientemente ou não; desejo, intento ${ }^{(12)}$, e Valor do Recurso Computacional, entendendo como valor ou conjunto de princípios ou normas a serem buscados pelo ser humano a fim de corporificar o ideal de perfeição ou plenitude moral ${ }^{(13)}$.

A pesquisa que originou este artigo foi aprovada pelo Comitê de Ética em Pesquisa da Pontifícia Universidade Católica do Paraná sob o parecer no 3309/2009. Após aceitarem participar da pesquisa, todos os docentes assinaram um termo de consentimento livre e esclarecido.

\section{RESULTADOS}

Foram extraídos 50 temas do corpus de análise, sendo 30 subcategorias relacionadas à categoria analítica Intenção e 20 subcategorias à categoria Valor. Após sucessivas leituras, este conjunto foi reagrupado em nove subcategorias interpretativas, sendo cinco relacionadas à Intenção: facilitar o processo de raciocínio clínico; possibilitar avaliação e autoavaliação do aluno; reflexão sobre a prática; facilitador da prática do ensino; e induzir mudança; e quatro ao Valor: utilidade para prática assistencial; inovação; aprendizagem e capacitação contínua.

\section{A intenção da inclusão de recurso computacional no ensino}

Em seus discursos, os docentes identificaram como uma intenção a capacidade de facilitar o processo de raciocínio clínico do aluno. Esta identificação se ancora nos depoimentos que apontam o recurso como organizador de dados, que aperfeiçoa a construção do raciocínio. Por outro lado, eles apontam uma limitação: grande parte dos enfermeiros dos campos de estágio não experimentou este tipo de recurso em sua formação, o que pode ser uma razão para desvalorização de métodos capazes de potencializar o raciocínio clínico. Fato que reflete no aprendizado do aluno, que não observa, tampouco presencia, a prática do raciocínio pelo enfermeiro. Também referem dificuldade para ensinar o aluno a raciocinar, por entenderem que isso faz parte do desenvolvimento do mesmo. Seus discursos indicam que uma das formas de facilitar este processo é a exposição de dificuldades:

Acho que o raciocínio diagnóstico não é uma coisa fácil do aluno fazer, mas a ferramenta dá essa possibilidade [...] de facilitar esse raciocínio. (P6)

Outra intenção emergida dos discursos é a do protótipo possibilitar avaliação e autoavaliação do aluno, como este recorte:

Se o aluno conseguir se autoavaliar, consegue reestruturar o seu conhecimento ou sua reflexão, faz uma análise crítica e faz uma nova proposta de ensino, seu próprio ensino. (P5)

A possível reflexão sobre a prática também foi levantada como intenção do protótipo. Os docentes relatam que nos campos de estágio, comumente, os alunos verificam ausência de registro dos elementos da prática de enfermagem. E, talvez, com a utilização rotineira de um recurso que possibilite o exercício do uso de linguagens próprias da profissão, este tema possa ser discutido com boa interação e consequente ganho para os hospitais universitários. Isso pode ser exemplificado pela seguinte frase temática:

Se bem que você percebe que às vezes essas etapas não se têm, mas com uma ferramenta facilitadora, acho que esta interação do enfermeiro seria muito diferente. (P6)

Nesta lógica, os docentes relatam que o protótipo pode ser utilizado também na educação continuada, mas é necessária a reflexão teórica dos conceitos referentes ao processo de raciocínio diagnóstico e a educação continuada. Apontam, ainda, que antes de utilizar algo novo, discutem com todos os envolvidos: 
Acho que a gente tinha que primeiro fazer um estudo teórico ou uma reflexão teórica da diferença entre esses conceitos. (P5)

Ao refletir sobre a aplicabilidade do protótipo, os docentes relatam que há uma intenção de que esse seja um facilitador da prática do ensino, mas reconhecem dificuldades no uso de recursos computacionais. As seguintes frases temáticas expõem a questão:

A informação da forma como é trazida, decodificada e oferecida, acho que pode ser uma ferramenta muito boa, muito facilitadora do nosso processo. (P1)

[...] também podem ser dificultadores no sentido de que muitas vezes temos que nos enquadrar na ferramenta. Então às vezes ela funciona como uma camisa de força. (P1)

A última subcategoria desvelada dos discursos dos docentes refere-se à intenção do protótipo como indutor de mudança. Pelo fato de recursos computacionais fazerem parte da vida do aluno, o protótipo foi identificado como capaz de conduzir o aluno a raciocinar, resolvendo um estudo de caso de maneira mais descontraída. Por outro lado, alguns professores levantaram a necessidade de uma discussão relacionada ao uso deste tipo de recurso, pois mesmo facilitando o seu trabalho, é premente avaliar o impacto desta mudança no ensino, ou seja:

Depois, para usar realmente no ensino, a gente tem que fazer esta discussão. (P5)

\section{Valores identificados no recurso computacional} para ensino

Os docentes destacaram como um dos valores do protótipo sua utilidade na prática assistencial, pela possibilidade de uso do sistema para além do espaço acadêmico:

Se for para agregar valor e qualidade a nossa prática, tem que ir para o campo, até para não ficar só aqui dentro. (P1)

Outro valor agregado ao protótipo diz respeito à inovação, que foi percebida de duas formas: como um estímulo para busca de novas metodologias e como um agregador de tecnologia para a prática profissional:
Acho que é uma relação de estímulo e de incentivo à busca de um novo conhecimento das novas metodologias. (P1)

O implícito talvez fosse de aproximar a prática, à nossa prática profissional com a tecnologia mais desenvolvida, que no primeiro momento a gente não percebe. $(\mathrm{P} 1)$

No que diz respeito ao valor identificado à aprendizagem, o discurso dos docentes aponta que a ferramenta trará qualidade ao aprendizado do raciocínio clínico, que está fortemente relacionada à sequência do método de ensino no qual foi baseado o protótipo. Também foi abordado que o recurso não substitui leituras diárias que devem ser feitas pelos alunos, mas que o mesmo pode agregar:

A ferramenta, nesse sentido, não que ela vá substituir leituras prévias. Ela não substitui, mas com certeza potencializa, chama mais atenção para que o aluno execute esse ato de ter um estudo um pouquinho mais rotineiro. (P4)

A capacitação contínua foi identificada como valor, sendo suporte institucional para conhecimento e posterior utilização do recurso computacional:

Tem que haver um bom treinamento, uma boa educação continuada, permanente, para esses profissionais e para os alunos da graduação. (P5)

\section{DISCUSSÃO}

Desde o início de 1990, a literatura já evidenciava que a condução do raciocínio clínico é um grande desafio no ensino da enfermagem e o exercício deste processo durante a graduação permite estreitar as possibilidades de erros ${ }^{(14)}$. O trabalho com didáticas computacionais pode inserir o aluno num processo de construção e elaboração do conhecimento, o que propiciará o raciocínio clínico frente às decisões necessárias nas práticas de campo, entretanto este processo depende do conteúdo trabalhado pelos docentes e da busca de soluções pelos alunos ${ }^{(7)}$.

Neste contexto, o protótipo analisado foi elaborado de forma a permitir a inserção de estudos de caso com variados graus de dificuldade. $O$ recurso permite verificar a realização da tarefa de cada aluno após o término do conteúdo proposto, assim como o aluno também pode conferir em qual fase do exercício ele 
teve maior dificuldade. De fato, resultados de um estudo $^{(15)}$ evidenciaram que alunos relatam a importância do reforço imediato das informações recebidas do professor, bem como a possibilidade de autoavaliação. Sabe-se que o processo avaliativo deve ocorrer ao longo do ensino-aprendizagem, bem como ser considerado um dos pilares da aprendizagem, ao permitir que professores e alunos percebam o quanto foi apreendido e quais as lacunas existentes no ensino, no aprendizado e na própria avaliação ${ }^{(16)}$. Verifica-se também que a inserção de tecnologias computacionais no ensino aprendizagem permite feedback mais frequente, rapidez na comunicação e acompanhamento do desempenho dos alunos $^{(15)}$. Entretanto, no que se refere à intenção do recurso, o discurso dos docentes aponta para a compreensão de que independente da intencionalidade estar explicitada, se o aluno não presenciar o processo de raciocínio na prática, ele concluirá que este é um aprendizado exclusivo da academia, distanciando a teoria da prática.

Um estudo concluiu que enfermeiros possuem conhecimento mínimo sobre a proposta da elaboração e do registro do DE, portanto sentem-se desconfortáveis em discutir esse tema ${ }^{(17)}$. Neste sentido, propiciar a discussão de estudos de caso com interação entre docentes, discentes e profissionais, pode ser estratégia para minimizar o desconforto e potencializar a elaboração e o efetivo registro do diagnóstico.

A utilização de um recurso computacional no ensino da enfermagem, ao garantir flexibilidade das informações, permite aprimorar técnicas de ensino ${ }^{(18)}$, facilitando a construção e solidificação de novos conhecimentos e favorecendo processo de raciocínio ${ }^{(5)}$. Entretanto, o discurso dos docentes discute que esta facilitação só será efetiva se o aluno possuir uma base sólida, alicerce que, por vezes, é construído por literatura subjetiva e abstrata, com pouca evidência.

A introdução do tema DE, tanto no ensino quanto na assistência, pode concentrar a atenção em normas pré-estabelecidas que se configurem em camisa de força ou num fascínio tecnológico que supere o pensar ${ }^{(17)}$. Soma-se a esta problemática, que ao incluir objetos educacionais digitais, os mesmos devem despertar no aluno curiosidade e criatividade, fazendo com que ele não deixe de estudar da maneira habitual simultaneamente ao uso da tecnologia ${ }^{(5)}$. Sendo assim, no aspecto da intenção de facilitar a prática do ensino, o discurso dos docentes reforça que o protótipo, como qualquer recurso pedagógico, não deve incluir todo conteúdo ao aluno, caso contrário, ele não desenvolverá o processo de raciocínio sem a ancoragem de um aparato tecnológico, ou seja, o aluno deve ser capaz de construir sua forma de pensar.

Verificam-se aspectos relevantes da utilização de recursos tecnológicos como instrumentos pedagógicos, entre eles, as mudanças para a melhoria da qualidade do processo de ensino-aprendizagem ${ }^{(19)}$. Paralelamente, exige professores bem formados com conhecimentos consistentes sobre didática e o desenvolvimento da ferramenta pedagógica que irá atender às necessidades de cada indivíduo e da coletividade ${ }^{(19)}$.

Além de representar novas oportunidades e desafios para educadores e estudantes, o uso de computador e seus recursos caracterizam uma nova forma de ensinare aprender, assim como, ao serem usados por enfermeiros assistenciais, os ambientes virtuais de aprendizagem são uma forma de potencializar a divulgação dos conhecimentos $^{(20-21)}$. Neste sentido, o protótipo analisado foi visto pelos docentes como instrumento de ensino que poderia auxiliar no desenvolvimento do raciocínio diagnóstico do enfermeiro, dando oportunidade para exercitar estudos de caso e discutilos com docentes e alunos durante a prática de campo.

Ao elaborar uma proposta educacional online, uma pesquisa demonstrou que o aluno pode explorar os conteúdos propostos, encontrando situações de tomada de decisão sobre os caminhos a serem seguidos e a própria prática do exercício da autonomia frente ao estudo ${ }^{(20)}$. Nesta lógica, docentes e alunos devem se preparar para essas novas possibilidades, construindo juntos conhecimentos e habilidades, que desencadearão um processo de raciocínio, que perdurará por toda sua vida profissional $^{(22)}$.

Discute-se, também, que tecnologias computacionais que permitem a interatividade, a comunicação bilateral e multidimensional, como as que são proporcionadas por grupos de discussão, proporcionam a construção do conhecimento, pois estabelecem a possibilidade de sanar dúvidas no próprio grupo ${ }^{(23)}$. $\mathrm{O}$ protótipo analisado neste estudo, ao não sugerir grupo de discussão, não será capaz de superar a relação dual docente-aluno e nos discursos dos docentes não foi identificada sugestão de outro recurso desta natureza. Cabe então, ao docente, propiciar espaço na organização do método pedagógico adotado, para que isso ocorra além do ambiente computacional.

Para que o ensino-aprendizagem do processo de enfermagem seja efetivo é reconhecido que os alunos devem aprimorar os conhecimentos e desenvolver habilidades observando e analisando os assuntos aborda- 
dos, ou seja, devem procurar apreender utilizando-se de diversos recursos pedagógicos ${ }^{(24)}$. Então, parece que o valor dado à aprendizagem, que docentes identificaram no protótipo, não é diferente do que se verifica na prática de ensino sem a inclusão de recursos computacionais.

Por fim, os discursos analisados corroboram com a necessidade de apoio técnico e pedagógico na utilização de recursos tecnológicos que, por sua vez, instiga a curiosidade e motiva a constante renovação e capacitação de todos os que estão envolvidos direta ou indiretamente com o uso de ferramentas computacionais ${ }^{(18,25-26)}$.

\section{CONCLUSÃO}

Ao responder a necessidade da reflexão sobre as intenções e valores incorporados na inclusão de tecnologias computacionais para o ensino do desenvolvimento do raciocínio, este estudo verificou que docentes de enfermagem compreendem que independentemente da intencionalidade, é premente que o aluno vivencie na prática de campo o exercício do raciocínio. No discurso analisado verificou-se que mesmo construído para uso no espaço acadêmico, o protótipo poderá ser utilizado na educação continuada, propiciando interação do serviço com a academia, o que pode contribuir para superação do distanciamento entre teoria e prática.

Conclui-se, pelos discursos dos docentes, que tecnologias computacionais de auxílio ao ensino do raciocínio não devem criar dependência de aparato tecnológico no aluno, e que o mesmo deve ser exposto a outros recursos pedagógicos que propiciem a autoavaliação e o aprendizado pela identificação de suas próprias limitações.

Também foi analisado que o valor da inovação identificado no protótipo é um estímulo para que o docente busque novas tecnologias para sua prática, e que o valor dado à aprendizagem não é diferente daquele oferecido à mesma, quando não se faz uso de tecnologia computacional.

Por fim, deve ser pensada a incorporação de espaços de discussão e avaliação do impacto da inclusão de recursos como o analisado na mudança da prática de ensino.

\section{REFERÊNCIAS}

1. Fochiera F, Vieira CS. O diagnóstico de enfermagem no contexto das ações de enfermagem: percepções dos enfermeiros docentes e assistenciais. Rev. Eletr. Enferm. [Internet] 2004;6(2) [acesso em 05 set 2011]. Disponível: http://www.fen.ufg.br/revista/revista6_2/diag.html
2. Cruz DALM, Pimenta CAM. Prática baseada em evidências, aplicada ao raciocínio diagnóstico. Rev. Latino-Am. Enfermagem [Internet] 2005;13(3) [acesso em 18 jul 2013]. Disponível: http://dx.doi.org/10.1590/ S0104-11692005000300017

3. Rodrigues RM. Relato de experiência na utilização do portfólio na graduação em enfermagem. Cogitare enferm. [Internet] 2012;17(4) [acesso em 18 jul 2013]. Disponível: http://ojs.c3sl.ufpr.br/ojs2/index.php/ cogitare/article/viewFile/30391/19666

4. Peres HLC, Meira KC, Leite MMJ. Ensino de didática em enfermagem mediado pelo computador: avaliação discente. Rev Esc Enferm USP [Internet] 2007;41(2) [acesso em 18 jul 2013]. Disponível: http://dx.doi. org/10.1590/S0080-62342007000200014

5. Cogo ALP, Pedro ENR, Silva APSS, Schatkoski AM, Catalan VM, Alves RHK. Objetos educacionais digitais em enfermagem: avaliação por docentes de um curso de graduação. Rev Esc Enferm USP [Internet] 2009;43(2) [acesso em 18 jul 2013]. Disponível: http://dx.doi. org/10.1590/S0080-62342009000200006

6. Lopes ACC, Ferreira AA, Fernandes JAL, Morita ABPS, Poveda VB, Souza AJS. Construção e avaliação de software educacional sobre cateterismo urinário de demora. Rev Esc Enferm USP [Internet] 2011;45(1) [acesso em 18 jul 2013]. Disponível: http://dx.doi. org/10.1590/S0080-62342011000100030

7. Melo FNP, Damasceno MMC. A construção de um software educativo sobre ausculta dos sons respiratórios. Rev Esc Enferm USP [Internet] 2006;40(4) [acesso em 18 jul 2013]. Disponível: http://www.ee.usp.br/reeusp/ upload/pdf/290.pdf

8. Gonçalves GR, Peres HHC, Rodrigues RC, Tronchin DMR, Pereira IM. Proposta educacional virtual sobre atendimento da ressuscitação cardiopulmonar no recém-nascido. Rev Esc Enferm USP [Internet] 2010;44(2) [acesso em 18 jul 2013]. Disponível: http:// dx.doi.org/10.1590/S0080-62342010000200025

9. Peres HHC, Kurcgant P. O ser docente de enfermagem frente a informática. Rev. Latino-Am. Enfermagem [Internet] 2004;12(1) [acesso em 18 jul 2013]. Disponível: http://dx.doi.org/10.1590/S0104-11692004000100014

10. Cubas MC, Albuquerque LM. O uso da classificação 
internacional para as práticas de enfermagem (CIPE®) e do inventário vocabular da classificação internacional das práticas de enfermagem em saúde coletiva (CIPESC $®$ ) como instrumento da consulta de enfermagem. In: Santos AS, Cubas MR, organizadores. Saúde coletiva linhas de cuidado e consulta de enfermagem. Rio de janeiro: Elsevier; 2012. p.77-94.

11. Fiorin JL. Elementos da análise de discurso. São Paulo: Contexto; 2005.

12. Houaiss A, Villar, MS. Dicionário Houaiss da língua portuguesa. $1^{a}$ ed. Rio de Janeiro: Objetiva; 2009. Intenção; p.1095.

13. Houaiss A, Villar, MS. Dicionário Houaiss da língua portuguesa. 1 ${ }^{a}$ ed. Rio de Janeiro: Objetiva; 2009. p.1920.

14. Cruz DALM, Pimenta CAM. Avaliação do doente com dor crônica em consulta de enfermagem: proposta de instrumento segundo diagnósticos de enfermagem. Rev. Latino-Am. Enfermagem. [Internet] 1999;7(3) [acesso em 18 jul 2013]. Disponível: http://dx.doi.org/10.1590/ S0104-11691999000300008

15. Cobb KL, Billings DM, Mays RM, Canty-Mitchell J. Peer review of teaching in web-based courses in nursing. Nurse Educ. 2001;26(6):274-9.

16. Both IJ. Ensinar e avaliar são de domínio público: resta saber se ensinar avaliando e avaliar ensinando também o são. Rev. HISTEDBR on-line [Internet] 2005 [acesso em 30 set Disponível: http://www.histedbr.fae.unicamp. br/revista/revis/revis18/art06_18.pdf.

17. Almeida MA. Competências e o processo ensinoaprendizagem do diagnóstico de enfermagem. Rev. bras. enferm. [Internet] 2004;57(3) [acesso em 18 jul 2013]. Disponível: http://dx.doi.org/10.1590/S003471672004000300004

18. Aquino PS, Melo RP, Lopes MVO, Pinheiro AKB. Análise do conceito de tecnologia na enfermagem segundo o método evolucionário. Acta Paul. Enferm. [Internet] 2010;23(5) [acesso em 18 jul 2013]. Disponível: http://dx.doi.org/10.1590/S0103-21002010000500017

19. Silva FM. Aspectos relevantes das novas tecnologias aplicadas à educação e os desafios impostos para a atuação dos docentes. Akrópolis [Internet] 2003;11(2) [acesso em 18 jul 2013]. Disponível: http://revistas. unipar.br/akropolis/article/view/334/301

20. Costa JB, Peres HHC, Rogenski NMB, Baptista CMC. Proposta educacional on-line sobre úlcera por pressão para alunos e profissionais de enfermagem. Acta Paul. Enferm. [Internet] 2009;22(5) [acesso em 18 jul 2013]. Disponível: http://dx.doi.org/10.1590/S010321002009000500002

21. Alvarez AG, Sasso GTMD. Aplicação de objeto virtual de aprendizagem, para avaliação simulada de dor, em estudantes de enfermagem. Rev. Latino-Am. Enfermagem. [Internet] 2011;19(2) [acesso em 18 jul 2013]. Disponível: http://www.scielo.br/pdf/rlae/v19n2/pt_02

22. Silva APSS, Cogo ALP. Aprendizagem de punção venosa com objeto educacional digital no curso de graduação em enfermagem. Rev. Gaúcha Enferm. [Internet] 2007;28(2). [acesso em 18 jul 2013]. Disponível: http://seer.ufrgs.br/ RevistaGauchadeEnfermagem/article/view/3162/1733

23. Dal Pai D, Lautert L. Grupos de discussão virtual: uma proposta para o ensino em enfermagem. Rev Esc Enferm USP [Internet] 2007;41(3) [acesso em 18 jul 2013]. Disponível: http://www.lume.ufrgs.br/bitstream/ handle/10183/69668/000604266.pdf?sequence $=1$

24. Amante LN, Anders JC, Meirelles BHS, Padilha MI, Kletemberg DF. A interface entre o ensino do processo de enfermagem e sua aplicação na prática assistencial. Rev. Eletr. Enferm. [Internet] 2010;12(1) [acesso em 12 set 2011]. Disponível: http://www.revistas.ufg.br/index. $\mathrm{php} /$ fen/article/view/9538

25. Araújo VE, Witt RR. O ensino de enfermagem como espaço para o desenvolvimento de tecnologias de educação em saúde. Rev. Gaúcha Enferm. [Internet] 2006;27(1) [acesso em 18 jul 2013]. Disponível: http://www.lume.ufrgs.br/bitstream/ handle/10183/23546/000536831. pdf?sequence $=1$

26. Cogo ALP, Silveira DT, Pedro ENR, Tanaka RY, Catalan VM. Aprendizagem de sinais vitais utilizando objetos educacionais digitais: opinião de estudantes de enfermagem. Rev. Gaúcha Enferm. [Internet] 2010;31(3) [acesso em 18 jul 2013]. Disponível: http://dx.doi. org/10.1590/S1983-14472010000300005 